\title{
A 1.8-V operation RF CMOS Receiver Front-End for WLAN/MAN
}

\author{
Mi-young Lee ${ }^{1}$ and Young-Hun Lee ${ }^{2 *}$ \\ ${ }^{1}$ Dept. of Electronic Eng., Hannam University, Ojeong -dong, Daedeok-gu, Daejon, \\ 306-791, Korea.Phone: + 82-42-629-7395 \\ ${ }^{2 *}$ Dept. of Electronic Eng., Hannam University, Ojeong -dong, Daedeok-gu, Daejon \\ 306-791, KoreaPhone: +82-42-629-7565, \\ aphro95@hanmail.net,yhlee00@hnu.kr \\ *Corresponding Author
}

\begin{abstract}
This paper describes a CMOS direct-conversion receiver front-end for $5 \mathrm{GHz}$ wireless LAN/MAN. Sub-harmonic mixing is used for down-conversion to minimize the DC-offset due to LO-leakage. The residual DC-offset is cancelled by a digital-to-analog converter at the output of mixer. Band-gap reference generates required bias voltages and currents for each block. Serial-to-parallel interface (SPI) circuit is used to provide the various control signals such as gain-control of low-noise amplifier (LNA). To minimize the DC-offset due to LO selfmixing, sub-harmonic mixing is used for down-conversion. For quadrature down-conversion with sub-harmonic mixing, octa-phase $L O$ signals are generated by octa-phase shifting network. Implemented in a $0.18 \square m$ CMOS technology, the receiver dissipates $62 \mathrm{~mA}$ from a $1.8 \mathrm{~V}$ supply voltage and has $3.1 \mathrm{~dB}$ noise figure $(N F)$ and $-13 \mathrm{dBm}$ input third-order intercept point (iIP3).
\end{abstract}

Keywords: CMOS (Complementary Metal Oxide Semiconductor), Receiver Front-End, Cascode LNA, Mixer

\section{Introduction}

Among various kinds of wireless communication systems, wireless LAN/MAN is most popular for short range communications due to its high data rate. For low cost and low power implementation of WLAN terminal, fully-integrated CMOS RF transceiver is required for which low-IF or zero-IF (direct-conversion) architecture is most suited because the number of external components is minimized [1-3]. Low-IF architecture provides much higher immunity for DC-offset and flicker noise than direct-conversion architecture but high-level of matching between signal paths is required for sufficient image rejection [3]. The $5 \mathrm{GHz}$ WLAN/MAN standard, IEEE 802.11a, 802.16a, is employing OFDM where the first sub-carrier is not used and the channel bandwidth is wide [4]. Therefore, it is relatively immune to DC-offset and flicker noise and direct-conversion receiver architecture has been a popular choice [1-2].

In this paper, implemented in a 0.18um CMOS technology, a fully-integrated CMOS direct conversion receiver front-end is described for $5 \mathrm{GHz}$ WLAN/MAN applications.

The block diagram of the direct-conversion receiver for 5GH WLAN/MAN is shown in Figure 1. The low-noise amplifier (LNA) is inductively degenerated cascoded common source amplifier with differential input and output. The LNA has dual gain mode to relax the 
linearity requirement on down-conversion mixer. The LNA is switched to low-gain mode when large RF input signal is present. In order to minimize the DC-offset generation due to the LO self-mixing, sub-harmonic mixing is employed for direct down-conversion.

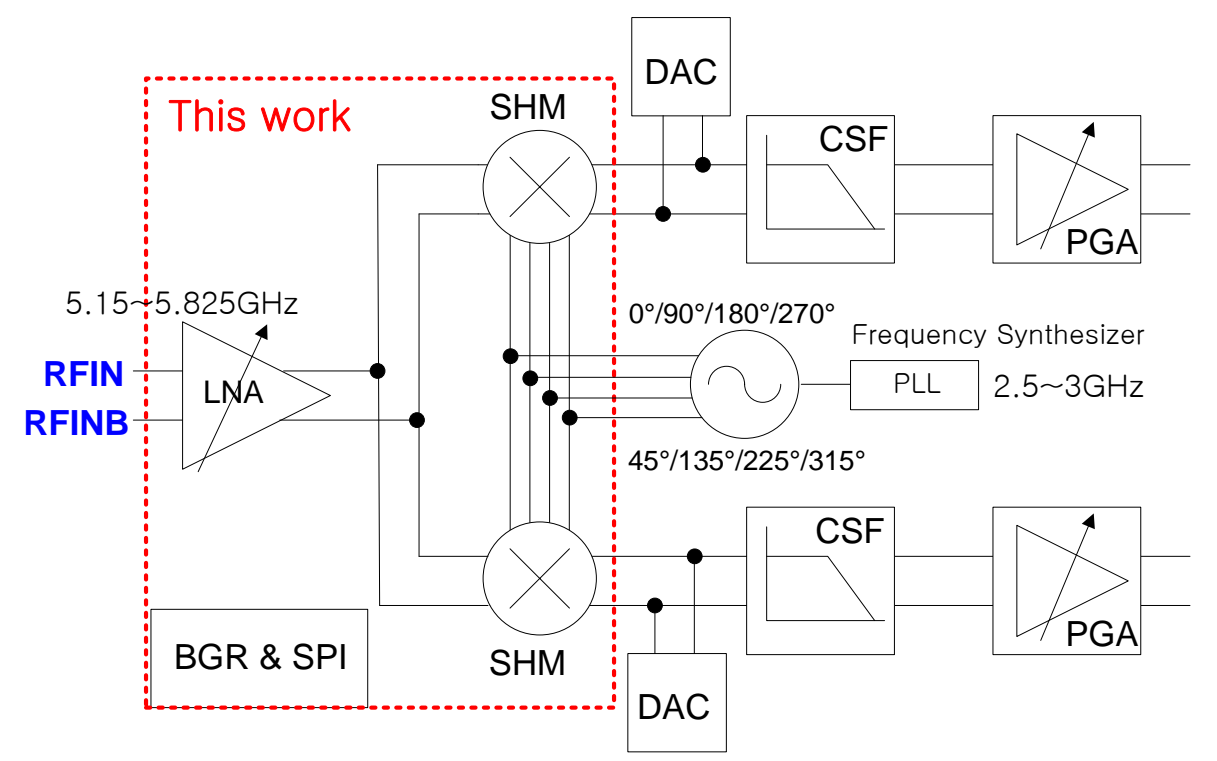

Figure 1. Direct-conversion receiver for 5GHz WLAN/MAN

\section{Related study}

The LNA is the first block in the receiver chain and is used to amplify the weak RF received signal. From a sensitivity standpoint, the noise figure of the LNA must be sufficiently low because its noise figure directly adds to the system noise figure. Also, its gain must be high enough to overcome the noise of the RF mixers and the subsequent stages. On the other hand, the linearity of the output of the RF mixers must be fairly high to accommodate the amplified input signal coming from the LNA. These two specifications elude to a tightly coupled design of both the LNA and the RF mixers. Another essential requirement of the RF front-end is to provide a good input match to the duplexer. This specification mandates a real $50 \Omega$ input resistance for the LNA. In addition to all of these requirements, it is desirable to minimize the NF and to maximize the IIP3 of these cells because they are continuously on during both High gain mode and Low gain mode. It provides signal amplification without degrading the signal-to-noise ratio too much.

\subsection{LNA structure}

Common-source LNA with inductive degeneration is widely used in many topologies. It achieves noise matching and power matching using components. This topology is shown in Figure 2. The input impedance can be expressed as

$$
\mathrm{Z}_{\mathrm{in}}=\frac{1}{j \omega C_{g s}}+j \omega\left(L_{g}+L_{s}\right)+\frac{g_{m} L_{s}}{C_{g s}}
$$


which contains a real term, $\frac{g_{m} L_{s}}{C_{g s}}$, that is proportional to the transit frequency $\omega_{T} \approx \frac{g_{m}}{C_{g s}}$. By properly choosing the dimensions of M1 and the value of $L_{s}$, the real term can be made equal to the $50 \Omega$ source resistance.

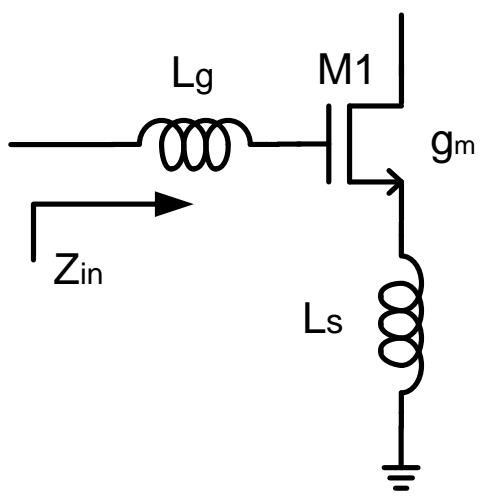

Figure 2. Common-source with inductive degeneration

Continuing the analysis with the above simplifications, the transconductance $G_{m}$ and noise factor $\mathrm{F}$ can be written as

$$
G_{m}=\frac{g_{m}}{\omega_{r} C_{g s}} \cdot \frac{1}{\left(R_{s}+\frac{L_{s} g_{m}}{C_{g s}}\right)}=g_{m} Q_{i n}=\frac{\omega_{T}}{\omega_{r}} \cdot \frac{1}{2 R_{s}}
$$

And $F=1+\gamma \frac{1}{g_{m} R_{s}} \cdot \frac{1}{\left(Q_{i n}\left(1+\frac{\omega_{T} L_{s}}{R_{s}}\right)\right)^{2}}=1+\gamma \frac{1}{g_{m} R_{s}} \cdot \frac{1}{4 Q_{i n}{ }^{2}}=1+\gamma g_{m} R_{s}\left(\frac{\omega_{r}}{\omega_{T}}\right)^{2}$

respectively, where $\gamma$ is the coefficient of thermal drain noise in a MOS transistor. Although the actual performance of the LNA deviates from the above simplified equations, these equations do give some general insight about the behavior of the LNA. Equation (2.3) shows that the noise figure of the LNA can be reduced by increasing $Q_{i n}$ [5]. This statement makes intuitive sense because at resonance, the gate-to-source voltage is equal to $Q_{i n}$ times the input voltage and thus the input voltage applied to transistor M1 is increased. Interestingly, for a common source amplifier, it can be shown that the optimum source impedance, in terms of noise, is an inductor that resonates with the input capacitance of the amplifier [6]. 


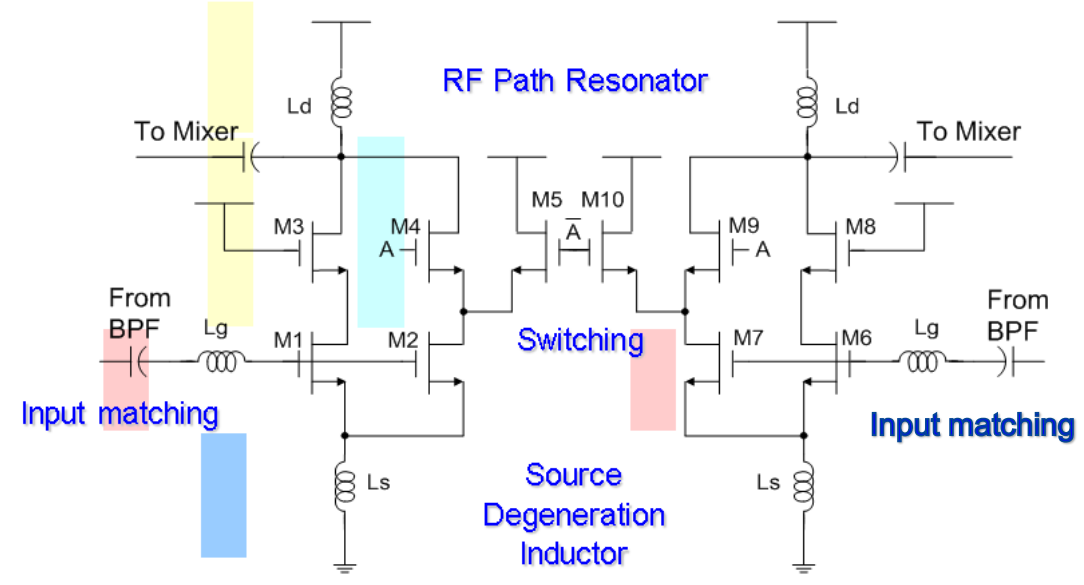

Figure 3. The schematic of a LC-tuned pseudo differential LNA

The proposed schematic of a LC-tuned pseudo differential LNA is shown in Figure 3. The LNA input is matched to the previous stage (e.g., output of an BPF). The LNA is based on the cascoded LNA structure with inductive degeneration and an inductive load. The pseudo differential low-noise amplifier uses the nMOS common-source cascode topology with inductive degeneration provided by bonding wire to minimize the chip size and to obtain a high Q-value, as shown in Figure 4. $L_{s}$ is used source degeneration inductor and $L_{g}$ is utilized with input impedance matching. The LNA has dual gain mode to relax the linearity requirement on down-conversion mixer. The LNA is switched to low gain mode to alleviate the linearity requirement on the following stages when large RF input signal is present. LCtuned pseudo differential LNA is used and two different voltage gain settings of 26 and $6 \mathrm{~dB}$ can be selected by switching the input transconductance. In high-gain mode, all the signal currents are directed to the output load network. In low-gain mode, the signal current generated by the transistor M2 and M7 is directed to VDD. The degeneration inductor $L_{s}$ is implemented using bonding wire.

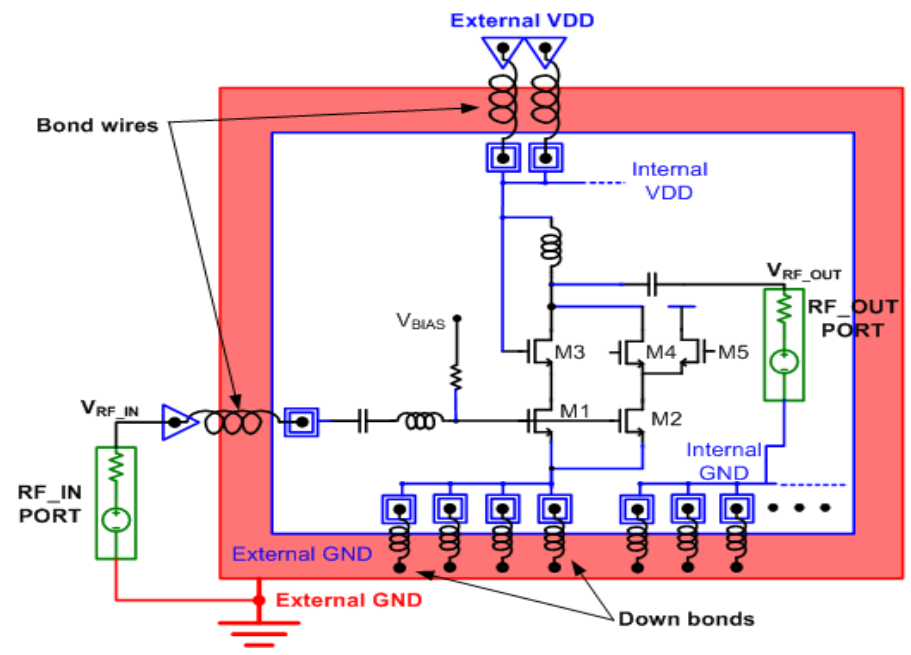

Figure 4. LNA structure using bond wires and down bonds for High Q-value 


\subsection{Sub-harmonic Mixer structure}

Figure 5 shows a simplified schematic of a single-balanced mixer with inductive loads. With a high intermediate frequency, inductive loads can be used in place of resistive loads to improve the filtering of the LO feed-through while increasing the head-room of the circuit. The gain of the mixer can be approximated by the following equation:

$$
A_{v} \approx \frac{2}{\pi} g_{m} R_{L}
$$

Where $R_{L}$ is the effective load resistance. The noise of the mixer can be estimated by the following simple but fairly accurate equation [7].

$$
\frac{\bar{v}_{o}}{\Delta f}=8 K T R_{L}\left(1+\gamma \frac{R_{L} I}{\pi A}+\frac{g_{m} R_{L}}{2}\right)
$$

where $I$ is the bias current of the input device and $A$ is the amplitude of the local oscillator. The first term is the effective noise of the two equivalent load resistors $R_{L}$, the second term is the output voltage noise from the switches M2 and M3, and the last term is the output voltage noise from the input transconductance device M1 assuming a conversion gain of $2 / \pi$. An interesting result of equation (2.5) is that the noise of the switches only depends on the local oscillator amplitude and bias current and is independent of the size of the switches as long as they turn on and off quickly [8]. To remove the DC-offset due to the LO self-mixing, the double-balanced sub-harmonic mixer shown in Figure 5 is used for quadrature downconversion. The frequency of the LO is the half of the RF and therefore eight-phase LO signals spaced by $45^{\circ}$ are required for quadrature down-conversion. As shown in the figure, the I-mixer uses $0^{\circ}, 90^{\circ}, 180^{\circ}$, and $270^{\circ} \mathrm{LO}$ signals while for Q-channel, $45^{\circ}, 135^{\circ}, 225^{\circ}$, and $315^{\circ}$ LO signals are used. The output of the transconductance stage is AC-coupled to the switching stage to prevent the low-frequency even-order harmonics and DC-offset of the transconductance stage from being leaked to the mixer output. Additional advantage of the AC-coupling is the independent biasing of the transconductance and switching stages, facilitating the design optimization such as the conversion gain, noise figure, and linearity. The output current of the mixer is converted to voltage by a first-order filter whose cut-off frequency is tuned by the same code as the channel selection filter following the mixer. 


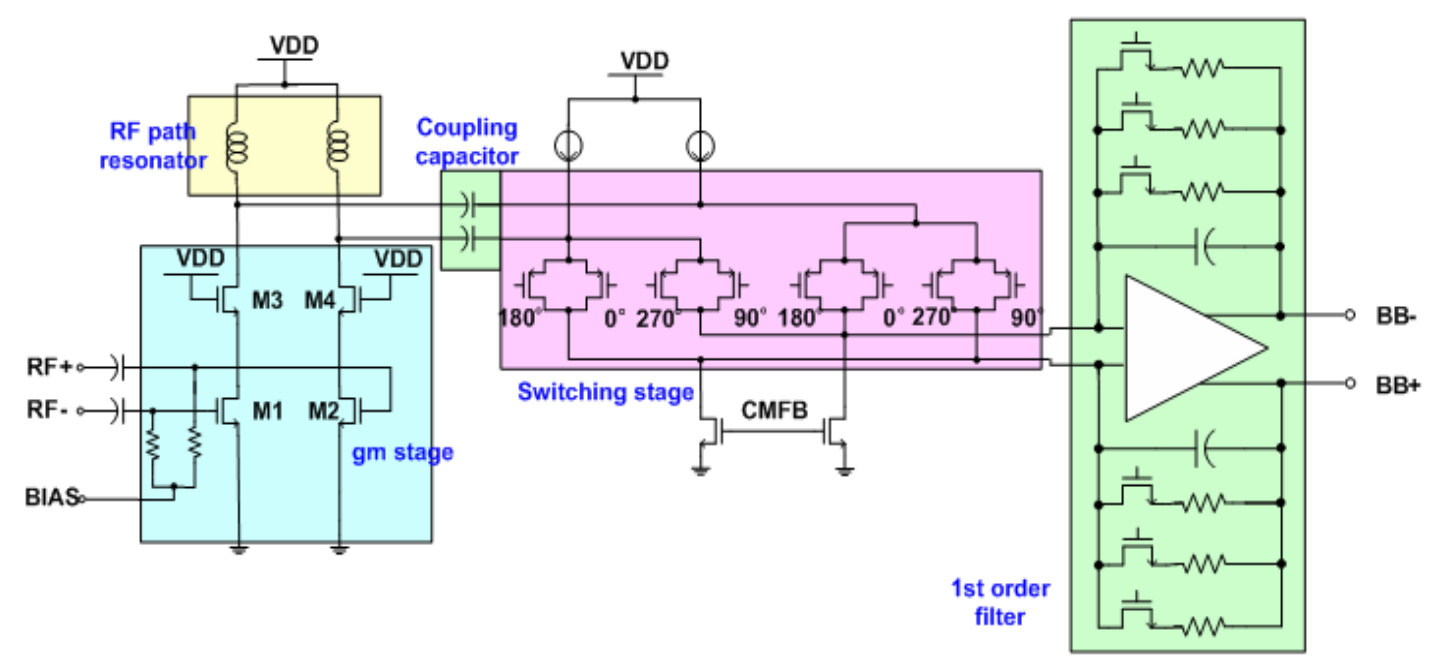

Figure 5. Sub-harmonic mixer for I-channel
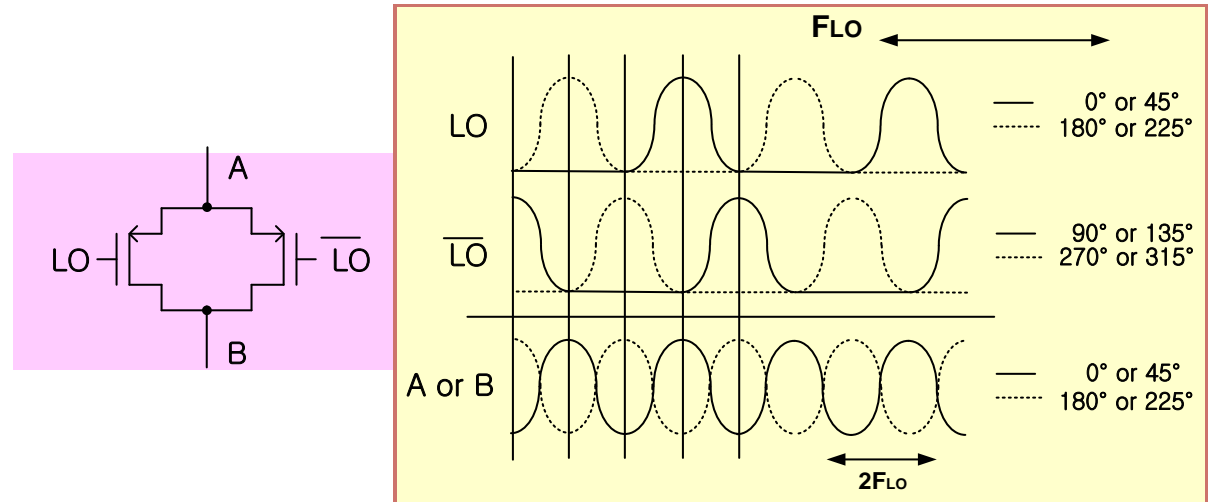

Figure 6. Operation principle of sub-harmonic mixing

For sub-harmonic mixing, the previous works normally used two stacked LO switching stages, requiring large voltage headroom $[9,10]$. In this work, only one stack of switching stage is used to allow the low-voltage operation and the principle of harmonic mixing is illustrated in Figure 6.

The conversion gain of the mixer is ;

$$
G=20 \log \left(\frac{2}{\pi} g_{m, R F} \cdot R_{\text {out }}\right)
$$

where $g_{m, R F}$ and $R_{\text {out }}$ are the transconductance of the RF input transistor and the output impedance, respectively. The input referred noise is given as ; 


$$
\overline{V_{n, i n}^{2}}=2 K T \pi^{2}\left[\frac{1}{g_{m, R F}^{2} R_{\text {out }}}+\frac{\gamma_{n}}{g_{m, R F}}+\frac{\gamma_{p}\left(g_{m, s w}+g_{m b, s w}\right)}{g_{m, R F}^{2}} \frac{T_{s w}}{T_{L O}}\right]+\overline{V_{n, \text { OPAMP }}^{2}}
$$

where $g_{m, s w}$ and $g_{m b, s w}$ are the transconductance and bulk transconductance of the switching transistors, respectively. $T_{L O}$ and $T_{s w}$ are the cycle time of LO and the duration when the two switching pairs are simultaneously turned on, respectively. The thermal noise of the switching transistors appears at the output during only $T_{s w}$.

From the equations (2.6) and (2.7), the conversion gain and the noise figure of the mixer can be improved by increasing the transconductance $g_{m, R F}$ of the RF input transistor. Larger $W / L$ ratio increases the parasitics and thereby the high-frequency operation, so the overdrive voltage is increased for higher $g_{m, R F}$. Higher overdrive voltage renders additional benefit of better linearity [11].

To minimize the noise contribution from the switching transistors, it is required to reduce the DC bias current of the switching stage and increase the LO amplitude as much as possible. In the proposed sub-harmonic mixer, the DC-bias of the switching stages is independent of the transconductance stage, allowing the simultaneously optimization of both the transconductance and switching stages. Moreover, the noise contribution of the switching stage is further reduced by resonating the parasitic capacitance at the node $A$ with the inductors $L_{1}$ and $L_{2}[12]$.

\section{An Experimental Result of WLAN/MAN Receiver Front-End}

The WLAN/MAN receiver front-end has been implemented in 0.18um CMOS RF technology whose microphotograph is shown in Figure 7. The chip occupies a core area of $2.1 \mathrm{~mm}^{2}$ and is packaged in a 48-pin MLF package with exposed die.

Measured voltage gain of the RF front-end is from $38.3 \mathrm{~dB}$ to $38.7 \mathrm{~dB}$ at high gain mode from 5.15 to $5.825 \mathrm{GHz}$ range as shown in Figure 8. Measured noise figure at LNA input is from $2.9 \mathrm{~dB}$ to $3.1 \mathrm{~dB}$ in the same frequency range as shown in Figure 9. To characterize the linearity performance of the receiver front-end, two in-band signals are applied to the system at $5.15 \mathrm{GHz}$ and $5.157 \mathrm{GHz}$, LO signal is applied $2.5675 \mathrm{GHz}$ and the power of the third order inter-modulation products measured for various input levels. The third-order input intercept point (IIP3) of the RF front-end the second-order input intercept point (IIP2) of the RF frontend is measured to be $-13 \mathrm{dBm}$ and $20 \mathrm{dBm}$, respectively, in LNA high-gain mode, as shown in Figures 10 and 11.

The results of the linearity, noise figure and voltage gain simulation and measurement of RF front-end are summarized in Table 1. with other performance parameters. 


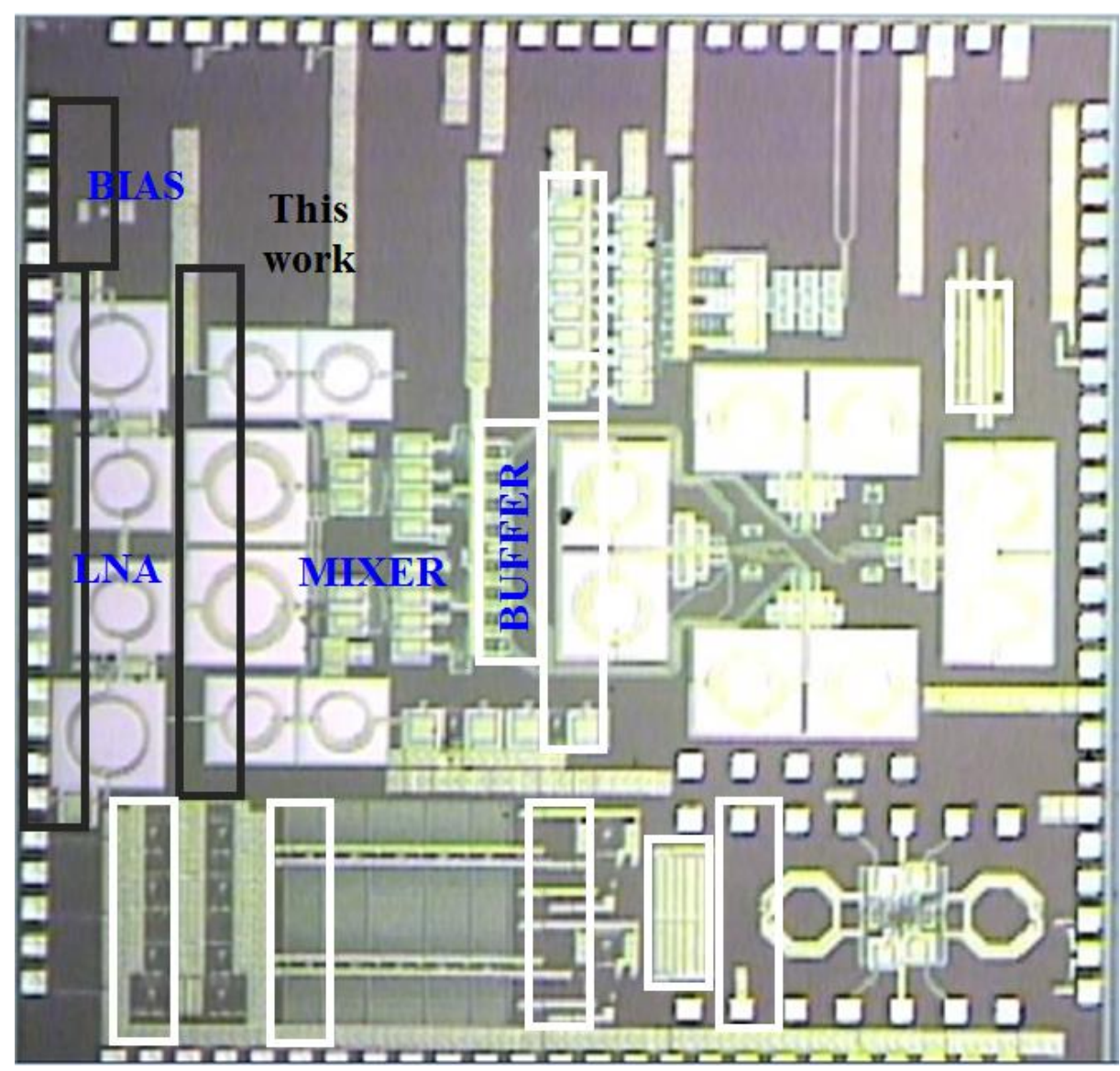

Figure 7. Die microphotograph of the receiver Front-End

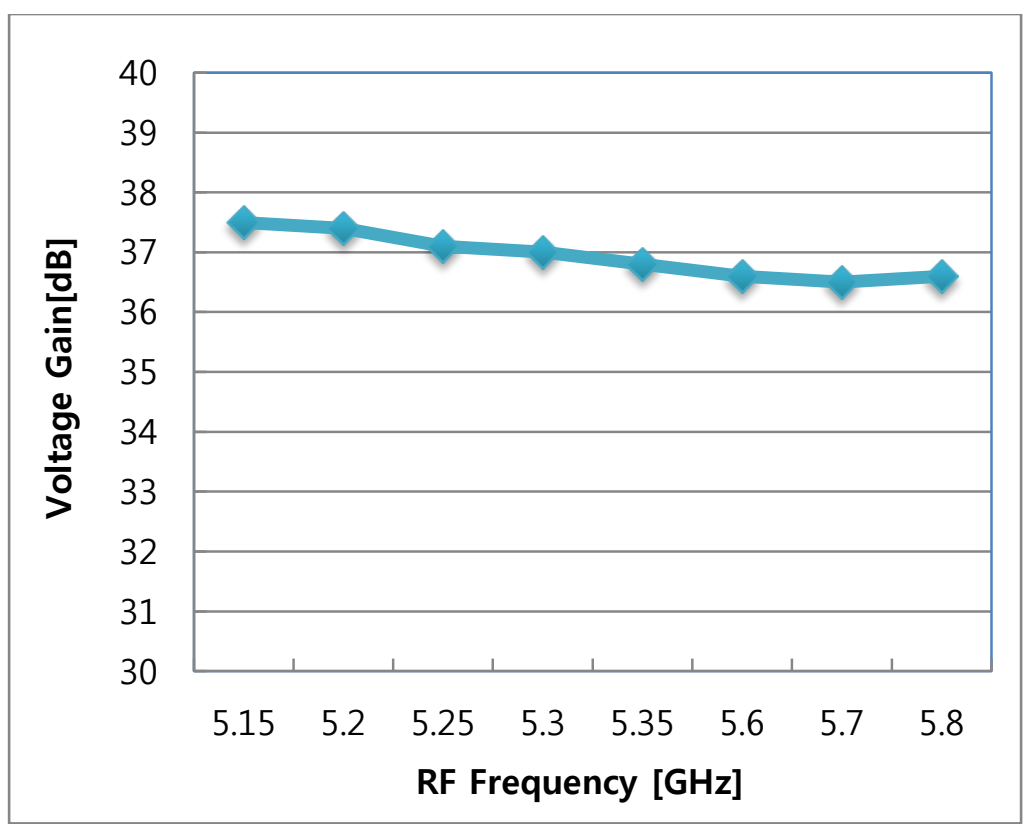

Figure 8. Measurement of front-end voltage gain at high gain mode 


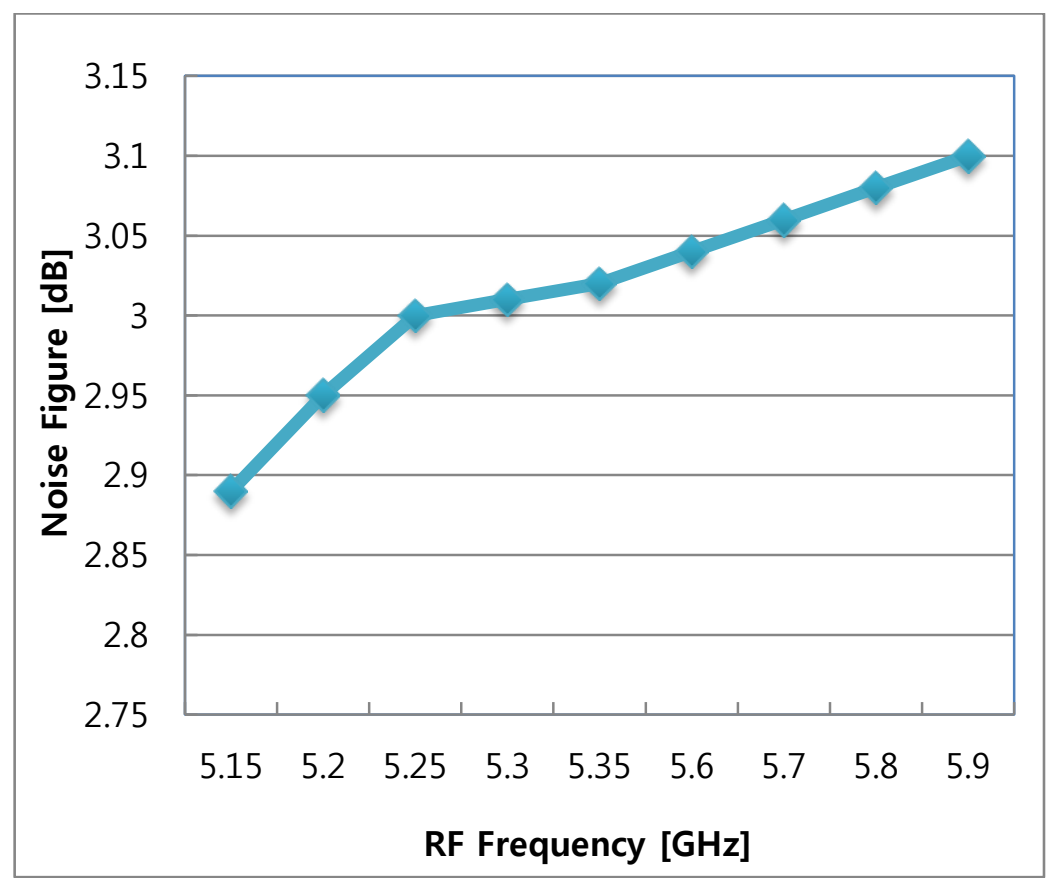

Figure 9. Measured Noise Figure at high gain mode

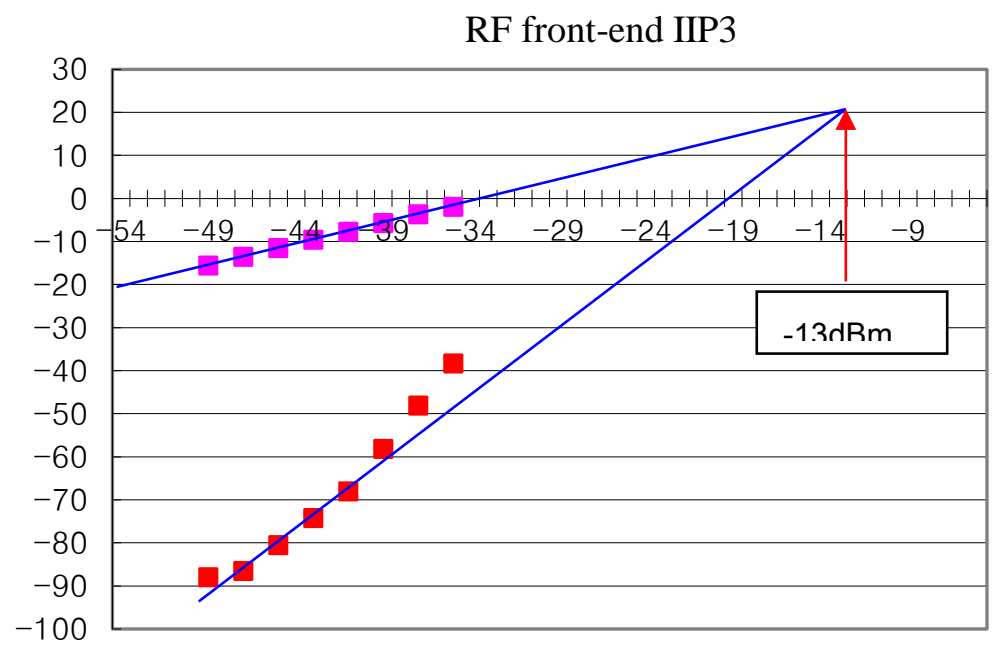

Figure 10. Measured IIP3 


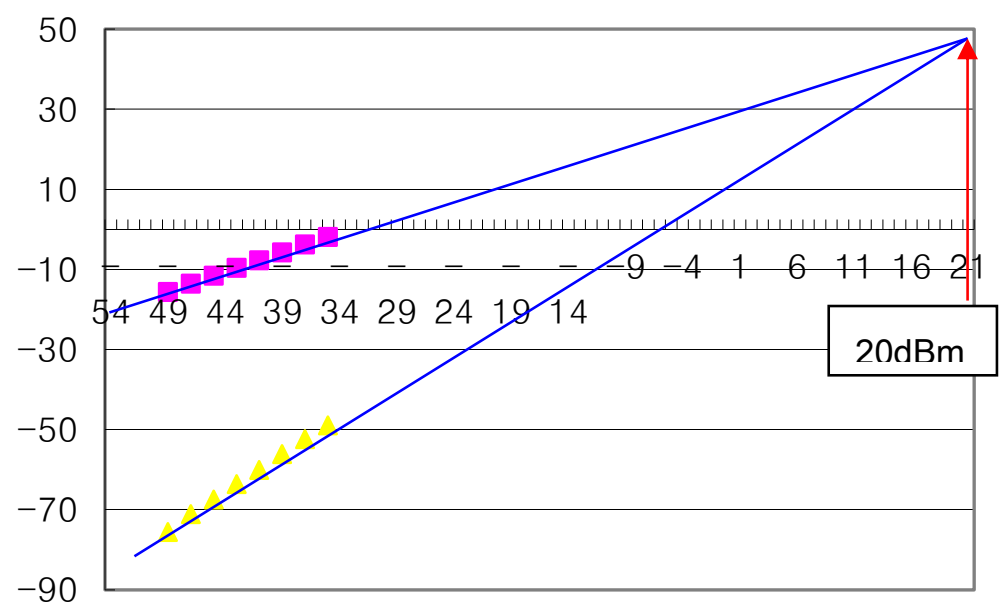

Figure 11. Measured IIP2

Table 1. Measured performance of RF Front-end

\begin{tabular}{cccc}
\hline Parameters & Spec. & Post simulation & Meas. Results \\
\hline Frequency $(\mathbf{G H z})$ & $5.15 \sim 5.825$ & $5.15 \sim 5.825$ & $5.15 \sim 5.825$ \\
\hline Voltage Gain[dB] & $28 \sim 38$ & 37.5 & $36.5 \sim 37.5$ \\
P1dB [dBm] & -22 & -22 & -21.4 \\
iIP3 [dBm] & -15 & -13.5 & -13 \\
Noise Figure[dB] & 3.2 & 2.9 & $2.88 \sim 3.1$ \\
\hline Power Cons.[mW] & 65 & 62.4 & 62.4 \\
\hline
\end{tabular}

\section{Conclusion}

This paper describes a CMOS direct-conversion receiver front-end for $5 \mathrm{GHz}$ wireless LAN/MAN. Sub-harmonic mixing is used for down-conversion to minimize the DC-offset due to LO-leakage. The residual DC-offset is cancelled by a digital-to-analog converter at the output of mixer. Band-gap reference generates required bias voltages and currents for each block. Serial-to-parallel interface (SPI) circuit is used to provide the various control signals such as gain-control of low-noise amplifier (LNA). To minimize the DC-offset due to LO self-mixing, sub-harmonic mixing is used for down-conversion. For quadrature downconversion with sub-harmonic mixing, octa-phase LO signals are generated by multi-phase shifting network. Implemented in a 0.18 um CMOS technology, the receiver dissipates $62 \mathrm{~mA}$ from a $1.8 \mathrm{~V}$ supply voltage and has $3.1 \mathrm{~dB}$ noise figure $(\mathrm{NF})$ and $-13 \mathrm{dBm}$ input third-order intercept point (iIP3).

\section{Acknowledgements}

This paper has been supported by 2013Hannam University Research Fund. 


\section{References}

[1] J. Vassiliou, K. Vavelidis, T. Georgantas, S. Plevridis, N. Haralabidis, G. Kamoulakos, C. Kapnistis, S. Kavadias, Y. Kokolakis, P. Merakos, J. C. Rudell, A. Yamanaka, S. Bouras and I. Bouras, "A single-chip digitally calibrated $5.12-5.825 \mathrm{GHz}$ 0.18um CMOS transceiver for 802.11a wireless LAN", IEEE J. SolidState Circuits, vol. 38, no. 12, (2003) December, pp. 2221-2231.

[2] P. Zhang, T. Nguyen, C. Lam, D. Gambetta, T. Soorapanth, B. Cheng, S. Hart, I. Sever, T. Bourdi, A. Tham and B. Razavi, "A 5GHz direct conversion CMOS transceiver", IEEE J. Solid-State Circuits, vol. 38, no. 12, (2003) December, pp. 2232-2238.

[3] B. Razavi, "RF Microelectronics", Prentice-Hall, (1998).

[4] IEEE Standard 802.11a-1999, Part 11: Wireless LAN Medium Access Control (MAC) and Physical Layer (PHY) Specifications.

[5] D.K. Shaeffer and T. H. Lee, “A 1.5-V, 1.5-GHz CMOS Low Noise Amplifier”, IEEE Journal of Solid-State Circuits, vol. 32, (1997) May, pp. 745-759.

[6] J. C. Rudell, et al., "A 1.9-GHz Wide-Band IF Double Conversion CMOS Receiver for Cordless Telephone Applications", IEEE Journal of Solid-State Circuits, vol. 32, (1997) December, pp. 2071-2088.

[7] A. A. Abidi, "High-Frequency Noise Measurements on FET's with Small Dimensions", IEEE Trans. Electron Devices, vol. ED-33, (1986) November, pp. 1801-1805.

[8] H. Darabi, "An Ultralow Power single-chip CMOS 900MHz Receiver for Wireless Paging", Ph.D. dissertation in Electrical Engineering, UCLA, (1999) June.

[9] L. E. Larson, "A wide-bandwidth SiGe HBT direct conversion sub-harmonic mixer/down-converter", IEEE J. Solid-State Circuits, vol. 35, no. 9, (2000) September.

[10] S. J. Fang, S. T. Lee and D. J. Allstot, "A 2GHz CMOS even harmonic mixer for direct conversion receivers", IEEE Int. Symp. Circuits and Systems, vol. 4, (2002), pp. IV-807-810.

[11] M.T Terrovitis and R. G. Meyer, "Intermodulation distorsion in current-commutating CMOS mixers", IEEE J. Solid-State Circuits, vol. 35, (2000) October, pp. 1461-1473.

[12] H. Darabi and A. A. Abidi, "Noise in RF-CMOS mixers: A simple physical model", IEEE J. Solid-State Circuits, vol. 35, (2000) January, pp. 15-25.

\section{Authors}

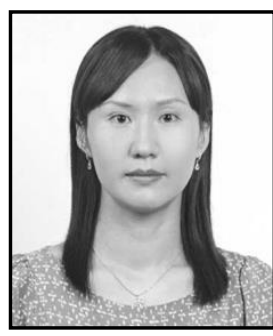

\section{Mi-myoung Lee}

2001. B.S. degree in Department of Information and Communication Engineering, Chonbuk University

2010. Ph.D. degrees in Department of Electronic and Computer Engineering, Hanyang University

2010-2011. Post-doctoral Fellowship in Department of Electrical and Electronics Engineering, KAIST 2011. Full time lecturer in Department of Electronics Engineering, Hannam University

2013. Assistant professor in Department of Electronics Engineering, Hannam University

Research interests: RFIC design, Analog and mixed mode IC design

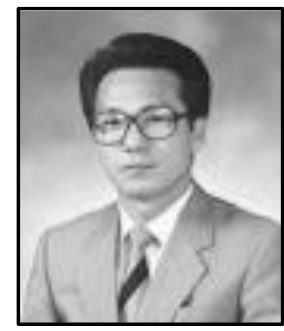

\section{Young-Hun Lee}

1973. Electronics Engineering, Donga University 1980. M.S. Electronics Engineering, Soongsil University 1984. Ph. D. Electronics Engineering, Kyoung Hee University Present. Professor in Electronics Engineering, Hannam University 
International Journal of Control and Automation Vol.6, No.6 (2013) 